\title{
Probability of Symbol Error for MPSK, MDPSK and Noncoherent MFSK with MRC and SC Space Diversity in Nakagami- $m$ Fading Channel
}

\author{
C. M. Lo and W. H. Lam \\ Department of Electrical and Electronic Engineering \\ The University of Hong Kong \\ Hong Kong, China
}

\begin{abstract}
This paper derives analytically some simple closed form expressions for the average symbol error rate (SER) of $M$-ary phase shift keying (MPSK), $M$-ary differential phase shift keying (MDPSK) and noncoherent $\boldsymbol{M}$-ary frequency shift keying (MFSK) over a Nakagami-m fading channel with $\boldsymbol{L}$ fold maximal ratio combining (MRC) and selection combining (SC) space diversity receptions. Numerical results demonstrate the error performance improvement by employing MRC and SC diversity receptions in the above communications systems and show that MRC improves the error performance more significantly than does simple SC.
\end{abstract}

\section{INTRODUCTION}

Over the years, compensation techniques for multipath channel fading in wireless communications have attracted much attention (see, e.g., [1-3] and references therein). Diversity combining, which skillfully combines multiple replicas of the received signals, has long been recognized as one of the effective compensation techniques for combating the detrimental effects of channel fading. Two of the methods to combine these multipath components are maximal ratio combining (MRC) and selection combining (SC) [1]. While MRC is known as the optimal combining technique by maximizing the signal-to-noise ratio (SNR) of the combined signal, $\mathrm{SC}$ is considered as the easiest method of combining multipath components by selecting the diversity branch with the largest SNR.

In the consideration of the mobile communications channels, Nakagami- $m$ distribution [4] is considered as one of the most versatile fading channel models. High level modulations are usually employed over these mobile channels since the bandwidth of them are very limited. Previous works [5] and [6] have evaluated the error performance of $M$-ary phase shift keying (MPSK) with $L$ fold MRC and SC, respectively over Nakagami- $m$ fading channel. Furthermore, [7] has evaluated the error performance of $M$-ary differential phase shift keying (MDPSK) with $L$-fold $S C$ over Nakagami- $m$ fading channel. The resulting error formulae given in these references are either in complicated closed form or in terms of an integral, which has to be evaluated numerically. Instead, this paper presents some simple closed form expressions of the average symbol error rate (SER) for MPSK and MDPSK with $L$-fold MRC and SC over
Nakagami- $m$ fading channel by using a close approximation for the conditional SER of MPSK and MDPSK. In addition, the analysis is extended to the case of noncoherent $M$-ary frequency shift keying (MFSK). These results are interesting since they can provide simple interpretations of these complicated communications systems.

This paper is organized as follows. Section II will describe the system model. The performance analyses of MRC and SC diversity receptions are presented in Section III and IV, respectively. Numerical results and some concluding remarks are presented in Section V and VI, respectively.

\section{SYSTEM MODEL}

Consider a communication system where the mobile communications fading channel is modeled as Nakagami-m distribution, the probability density function ( $\mathrm{pdf}$ ) of the instantaneous received SNR per symbol $\rho_{i}$ on the $i$ th branch of a receiver with diversity combining is given by [4]

$$
p\left(\rho_{i}\right)=\left(\frac{m}{\Omega}\right)^{m} \frac{\rho_{i}^{m-1}}{\Gamma(m)} \exp \left(-\frac{m}{\Omega} \rho_{i}\right)
$$

where $m$ is the fading severity parameter with values from 0.5 to $\infty, \Omega$ is the average SNR per symbol per branch, and $\Gamma($.) denotes the gamma function [8]. Note that all branches are assumed to have the same values of $m$ and $\Omega$. With the $L$-fold diversity combining implemented in the receiver and equal noise power in the branches, the instantaneous SNR per symbol $\gamma$ at the output of the receiver is given by $\gamma=\sum_{i=1}^{L} \rho_{i}$ for $\operatorname{MRC}$ and $\gamma=\max \left(\rho_{1}, \rho_{2}, \ldots, \rho_{L}\right)$ for SC. With these channel statistics, the average SER at the output of the receiver can then be calculated by averaging the conditional probability of error over the pdf of $\gamma$, i.e.

$\bar{P}=\int_{0}^{\infty} P_{e}(\gamma) p(\gamma) d \gamma$

where $P_{e}(\gamma)$ is the conditional probability of symbol error for a particular communications system on the assumption 
that $\gamma$ is known.

The classical conditional SERs for MPSK and MDPSK involve an integral which has to be evaluated numerically. However, two simple good approximations for the conditional SER for MPSK [9] and MDPSK [10] are employed here, and surprisingly, they are in the same mathematical form. Thus a simple unified expression can be employed in the performance analysis and is given by

$$
P_{e, P S K}(\gamma) \cong A \operatorname{erfc}(\sqrt{B \gamma})
$$

where $\operatorname{erfc}($.$) is the complementary error function,$

$$
A=\left\{\begin{array}{cc}
1, & M P S K \\
\sqrt{\frac{1+\cos (\pi / M)}{2 \cos (\pi / M)},} & M D P S K
\end{array}\right.
$$

and

$$
B=\left\{\begin{array}{ll}
\frac{1-\cos (2 \pi / M)}{2}, & M P S K \\
1-\cos (\pi / M), & M D P S K
\end{array} .\right.
$$

Although PSK theoretically outperforms FSK by $3 \mathrm{~dB}$, FSK is still desirable because of its simple and flexible signaling for multichannel communications. Therefore, it is of interest to investigate its error performance, and the well known conditional SER for noncoherent MFSK is [11]

$$
P_{e, F S K}(\gamma)=\frac{1}{M} \sum_{i=2}^{M}(-I)^{i}\left(\begin{array}{c}
M \\
i
\end{array}\right) \exp \left[-\left(1-\frac{1}{i}\right) \gamma\right]
$$

\section{ERROR PERFORMANCE WITH MRC}

With the use of $L$-fold MRC diversity reception for communications systems in Nakagami- $m$ fading channel, the pdf of the instantaneous SNR per symbol of the combined signal becomes [5]

$$
p_{M R C}(\gamma)=\left(\frac{m}{\Omega}\right)^{L m} \frac{\gamma^{L m-1}}{\Gamma(L m)} \exp \left(-\frac{m}{\Omega} \gamma\right)
$$

After a substitution of (6) and (5) into (2), the average SER of noncoherent MFSK with $L$-fold MRC in a Nakagami- $m$ fading channel can be written as

$$
\begin{aligned}
\vec{P}_{M R C, F S K}=\frac{1}{M} \sum_{i=2}^{M}(-1)^{i}\left(\begin{array}{c}
M \\
i
\end{array}\right)\left(\frac{m}{\Omega}\right)^{L m} \frac{L}{\Gamma(L m)} \\
\\
\quad \times \int_{0}^{\infty} \gamma^{L m-1} \exp \left[-\left(1-\frac{1}{i}+\frac{m}{\Omega}\right) \gamma\right] d \gamma .
\end{aligned}
$$

By recognizing that the integral in (7) is a simple Laplace Transform integral, one obtains the $\bar{P}_{M R C, F S K}$ as

$$
\bar{P}_{M R C, F S K}=\frac{1}{M}\left(\frac{m}{\Omega}\right)^{L m} \sum_{i=2}^{M}(-I)^{i}\left(\begin{array}{c}
M \\
i
\end{array}\right)\left(1-\frac{1}{i}+\frac{m}{\Omega}\right)^{-L m}
$$

In addition, substitution of (6) and (3) into (2) yields immediately the average SER of MPSK and MDPSK with $L$-fold MRC in Nakagami- $m$ fading channel given by

$$
\bar{P}_{M R C, P S K}=\left(\frac{m}{\Omega}\right)^{L m} \frac{A}{\Gamma(L m)} \int_{0}^{\infty} \gamma^{L m-1} \exp \left(-\frac{m}{\Omega} \gamma\right) \operatorname{erfc}(\sqrt{B \gamma}) d \gamma
$$

After further simplification with the use of a relation in [12], a simple closed form expression for $\bar{P}_{M R C, P S K}$ can be simplified to

$$
\bar{P}_{M R C, P S K}=2 A\left(\frac{1-\mu_{c}}{2}\right)^{L m} \sum_{k=0}^{L m-1}\left(\begin{array}{c}
L m-1+k \\
k
\end{array}\right)\left(\frac{1+\mu_{c}}{2}\right)^{k}
$$

where

$$
\mu_{c}=\sqrt{\frac{\Omega / m}{1 / B+\Omega / m}} .
$$

This simple expression is thus able to provide the average SER for both MPSK and MDPSK by simply changing the values of $A$ and $B$.

\section{ERROR PERFORMANCE WITH SC}

Considering a receiver implementing $L$-fold SC diversity reception, the pdf of the instantaneous SNR per symbol of the combined signal at the output of the receiver is given by [13]

$$
p_{S C}(\gamma)=\left(\frac{m}{\Omega}\right)^{m} \frac{L \gamma^{m-1}}{[\Gamma(m)]^{L}}\left\{\gamma\left(m, \frac{m \gamma}{\Omega}\right)\right\}^{L-1} \exp \left(-\frac{m}{\Omega} \gamma\right)
$$

where $\gamma(\alpha, \beta)$ is the incomplete gamma function given by

$\gamma(\alpha, \beta)=\int_{0}^{\beta} e^{-t} t^{\alpha-1} d t$

When (12) is employed in (2) to evaluate the error probability of a communications system with $L$-branch SC diversity reception, the resulting average SER from (2) cannot be expressed in closed form and the results have to be solved numerically. However, (12) can be written as in 
(14) for integer $m[7]$

$$
\begin{aligned}
p_{S C}(\gamma)= & \frac{L}{\Gamma(m)} \sum_{l=0}^{L-1}(-1)^{l}\left(\begin{array}{c}
L-1 \\
l
\end{array}\right) \exp \left(-(l+1) \frac{m}{\Omega} \gamma\right) \\
& \times \sum_{k=0}^{l(m-1)} b_{k}^{l}\left(\frac{m}{\Omega}\right)^{m+k} \gamma^{m+k-1}
\end{aligned}
$$

where

$b_{0}^{l}=1, \quad b_{1}^{l}=l, \quad b_{l(m-1)}^{l}=\frac{1}{(\Gamma(m))^{l}}$

$b_{k}^{l}=\frac{1}{k} \sum_{j=1}^{J_{0}} \frac{j(l+1)-k}{j !} b_{k-j}^{l}$

with $J_{0}=\min (k, m-1), 2 \leq k \leq l(m-1)-1$.

After substitution of (14) and (5) into (2), the average SER of noncoherent MFSK with $L$-fold SC in Nakagami- $m$ fading channel can be expressed as

$$
\begin{aligned}
\bar{P}_{S C, F S K}= & \frac{1}{M} \sum_{i=2}^{M}(-1)^{i}\left(\begin{array}{c}
M \\
i
\end{array}\right) \frac{1}{\Gamma(m)} \sum_{l=0}^{L-1}(-1)^{l}\left(\begin{array}{c}
L-1 \\
l
\end{array}\right) \sum_{k=0}^{l(m-1)} b_{k}^{l}\left(\frac{m}{\Omega}\right)^{m+k} \\
& \times \int_{0}^{\infty} \gamma^{m+k-1} \exp \left[-\left(1-\frac{1}{i}\right) \gamma-(l+1) \frac{m}{\Omega} \gamma\right] d \gamma
\end{aligned}
$$

The integral in (16) is again a Laplace Transform integral, and $\bar{P}_{S C, F S K}$ finally can thus be simplified as

$$
\begin{aligned}
\bar{P}_{S C, F S K}=\frac{L}{M} \sum_{i=2}^{M} & \sum_{l=0}^{L-1} \sum_{k=0}^{l(m-1)}(-1)^{i+l}\left(\begin{array}{c}
M \\
i
\end{array}\right)\left(\begin{array}{c}
L-1 \\
l
\end{array}\right) b_{k}^{l} \\
& \times(m)_{k}\left(\frac{m / \Omega}{1-1 / i+(l+1) m / \Omega}\right)^{m+k}
\end{aligned}
$$

where $(a)_{b}$ is the Pochhammer's symbol [8]. In addition, the average SER of MPSK and MDPSK with $L$-fold SC in Nakagami-m fading channel can be obtained by substituting (14) and (3) into (2) as shown below

$$
\begin{aligned}
\bar{P}_{S C, P S K} & =\frac{A L}{\Gamma(m)} \sum_{l=0}^{L-1}(-l)^{l}\left(\begin{array}{c}
L-1 \\
l
\end{array}\right)^{l(m-1)} \sum_{k=0}^{l}\left(\frac{m}{\Omega}\right)^{m+k} \\
& \times \int_{0}^{\infty} \gamma^{m+k-1} \exp \left[-(l+1) \frac{m}{\Omega} \gamma\right] \operatorname{erfc}(\sqrt{B \gamma}) d \gamma .
\end{aligned}
$$

Further simplification yields the final simple expression for $\bar{P}_{S C, P S K}$ as

$$
\begin{aligned}
\bar{P}_{S C, P S K} & =\frac{2 A L}{\Gamma(m)} \sum_{l=0}^{L-1} \sum_{k=0}^{l(m-1)} \sum_{n=0}^{m+k-1}(-1)^{l}\left(\begin{array}{c}
L-1 \\
l
\end{array}\right) b_{k}^{l} \\
& \times \frac{(m+k-1)_{n}}{(l+1)^{m+k}}\left(\frac{1-\mu_{d}}{2}\right)^{m+k}\left(\frac{1+\mu_{d}}{2}\right)^{n}
\end{aligned}
$$

where

$$
\mu_{d}=\sqrt{\frac{\Omega / m(l+1)}{1 / B+\Omega / m(l+1)}} .
$$

Furthermore, it is also of interest to investigate the error performance of these three modulation schemes with $L$ fold SC in Nakagami- $m$ fading channel when $m$ is not an integer. However, closed form expression for average SER in this case is only possible for the case of dual SC diversity systems. Therefore, the average SER of these three modulation schemes with dual SC diversity over a Nakagami- $m$ fading channel are discussed here. The average SER of noncoherent MFSK in this situation can then be evaluated by substituting (12) with $L=2$ and (5) into (2) as

$$
\begin{aligned}
\bar{P}_{S C 2, F S K} & =\frac{1}{M} \sum_{i=2}^{M}(-1)^{i}\left(\begin{array}{c}
M \\
i
\end{array}\right)\left(\frac{m}{\Omega}\right)^{m} \frac{2}{[\Gamma(m)]^{2}} \\
& \times \int_{0}^{\infty} \gamma^{m-1} \exp \left[-\left(1-\frac{1}{i}+\frac{m}{\Omega}\right) \gamma\right] \gamma\left(m, \frac{m}{\Omega} \gamma\right) d \gamma .
\end{aligned}
$$

With the use of a relation in [8], (21) can be represented in terms of hypergeometric function and be simplified to

$$
\begin{aligned}
& \bar{P}_{S C 2, F S K}=\frac{2}{M} \sum_{i=2}^{M}(-I)^{i}\left(\begin{array}{c}
M \\
i
\end{array}\right)\left(\begin{array}{c}
2 m-1 \\
m
\end{array}\right)\left(\frac{m}{\Omega}\right)^{m+2} \\
& \times\left(1-\frac{1}{i}+\frac{2 m}{\Omega}\right)^{-2 m}{ }_{2} F_{1}\left(1,2 m ; m+1 ; \frac{m}{(2 m+\Omega) i-\Omega}\right)
\end{aligned}
$$

where ${ }_{2} F_{1}(a, b ; c ; x)$ is Gauss hypergeometric function[8].

For the cases with MPSK and MDPSK, the average SER with dual SC diversity over a Nakagami- $m$ fading channel can be calculated by substituting (12) with $L=2$ and (3) into (2) as

$$
\begin{aligned}
& \bar{P}_{S C 2, P S K}=\frac{2 A}{[\Gamma(m)]^{2}}\left(\frac{m}{\Omega}\right)^{m} \\
& \quad \times \int_{0}^{\infty} \gamma^{m-1} \exp \left(-\frac{m}{\Omega} \gamma\right) \operatorname{erfc}(\sqrt{B \gamma}) \gamma\left(m, \frac{m}{\Omega} \gamma\right) d \gamma .
\end{aligned}
$$


With the use of a summation representation of the incomplete gamma function as

$\gamma(\alpha, \beta)=\sum_{n=0}^{\infty} \frac{(-1)^{n}}{n !(\alpha+n)}(\beta)^{\alpha+n}$

the final closed form expression of $\bar{P}_{S C 2, P S K}$ can then be written as

$$
\begin{gathered}
\bar{P}_{S C 2, P S K=}=\frac{4 A}{[\Gamma(m)]^{2}} \sum_{n=0}^{\infty} \sum_{k=0}^{2 m+n-1} \frac{(-1)^{n}(2 m+n-1)_{k}}{n !(m+n)} \\
\times\left(\frac{1-\mu_{c}}{2}\right)^{2 m+n}\left(\frac{1+\mu_{c}}{2}\right)^{k}
\end{gathered}
$$

where $\mu_{c}$ is given as in (11).

\section{NUMERICAL RESULTS}

By using the simple closed form expressions in (8), (10), (17) and (19), the numerical values of the average SER for the three $M$-ary modulations (all $M=4$ ) with $L=2,3$ and 4 diversity branches are plotted in Fig. 1 to 6 . Fig. 1 and 2 show the average SER of noncoherent MFSK with both MRC and SC diversity receptions in Nakagami- $m$ fading channel for $m=5$ and $m=10$ respectively. Fig. 3 and 4 show the corresponding numerical values for MPSK, and the cases for MDPSK are shown in Fig. 5 and 6.

As for the case of noncoherent MFSK in Fig. 2, the required average SNR per symbol for an average SER of $10^{-6}$ are $13.2 \mathrm{~dB}, 11 \mathrm{~dB}$ and $9.4 \mathrm{~dB}$ for $L=2,3,4 \mathrm{MRC}$ diversity receptions. The corresponding values for $\mathrm{SC}$ diversity receptions are $15.7 \mathrm{~dB}, 14.9 \mathrm{~dB}$ and $14.4 \mathrm{~dB}$. As expected, the performance improvement of employing $\mathrm{MRC}$ is greater than SC for the same number of diversity branches. Observed from these numerical values, an increase of $L$ from 2 to 3 rendered more performance improvement than from 3 to 4 . It indicates that the performance improvement is somewhat retained when additional diversity branch is employed to an existing high number of diversity branches system. One final observation is that an additional diversity branch in MRC has much more improvement than does in SC when both have the same number of branches. Thus, the performance improvement by employing MRC is even more significant than $\mathrm{SC}$ when $L$ is large. Similar conclusions can be drawn from all other figures.

\section{CONCLUSIONS}

This paper derived some simple closed form expressions for the average SER of MPSK, MDPSK and noncoherent MFSK with $L$-fold MRC and SC (integer $m$ ) diversity receptions in a Nakagami- $m$ fading channel. For the case of arbitrary values of $m$, simple closed form on average SER expressions for the three $M$-ary modulations are also derived for the case of dual SC diversity reception. Results show that MRC performs significantly better than SC and this effect is more pronounced when the number of diversity branches is large.

\section{REFERENCES}

[1] M. Schwartz, W.R. Bennett and S. Stein, Communication systems and techniques, New York: McGraw-Hill, 1966.

[2] Yoshiya Miyagaki, Norihiko Morinaga and Toshihiko Namekawa, "Error probability characteristics for CPSK signal through m-distributed fading channel," IEEE Trans. Commun., vol. 26, no. 1, pp. 88-99, January 1978.

[3] Adnan A. Abu-Dayya and Norman C. Beaulieu, "Micro- and macrodiversity NCFSK (DPSK) on shadowed nakagamifading channels," IEEE Trans. Commun., vol. 42, no. 9, pp. 2693-2702, September 1994.

[4] M. Nakagami, "The m-distribution - A general formula of intensity distribution of rapid fading," in Statistical Methods of Radio Wave Propagation, W.C. Hoffman Ed., New York: Pergamon Press, pp. 3-26, 1960.

[5] V. Aalo and S. Pattaramalai, "Average error rate for coherent MPSK signals in nakagami fading channels," IEE Electronics Letters, vol. 32, no. 17, pp. 1538-1539, 1996.

[6] S.J. Baik, S.Y.Choi, D.W. Yoon, K.J. Lee and Y.Y. Han, "Analysis of selection diversity for MPSK signals in Nakagami fading channels," IEEE ICPWC, pp. 138-141. 1997.

[7] Gennaro Fedele, "N-branch diversity reception of M-ary dpsk signals in slow and nonselective Nakagami fading," Eur. Trans. Telecommun., vol. 7, no. 2, pp. 119-123, MarchApril 1996.

[8] 1. Gradshteyn and I. Ryzhik, Tables of integrals, series and products. New York: Academic, 1980.

[9] R. Knopp and H. Leib, "M-ary phase coding for the noncoherent AWGN channel," IEEE Trans. Inform. Theory, vol. 40, no. 6, pp. 1968-1984, November 1994.

[10] R.F. Pawula, "Asympotics and error rate bounds for M-ary DPSK," IEEE Trans. Commun., vol. 32, no. 1, pp. 93-94, January 1984.

[11] J. Sun and I.S. Reed, "Performance of MDPSK, MPSK and noncoherent MFSK in wireless Rician fading channels," IEEE Trans. Commun., vol. 47, no. 6, pp. 813-816, June 1999.

[12] J.G. Proakis, Digital Communications, $3^{\text {rd }}$ edition, New York: McGraw-Hill, 1995.

[13] M.S. Alouini and M.K. Simon, "Performance of coherent receivers with hybrid SC/MRC over Nakagami-m fading channels," IEEE Trans. Veh. Technol., vol. 48, no. 4, pp. $1155-1164$, July 1999. 


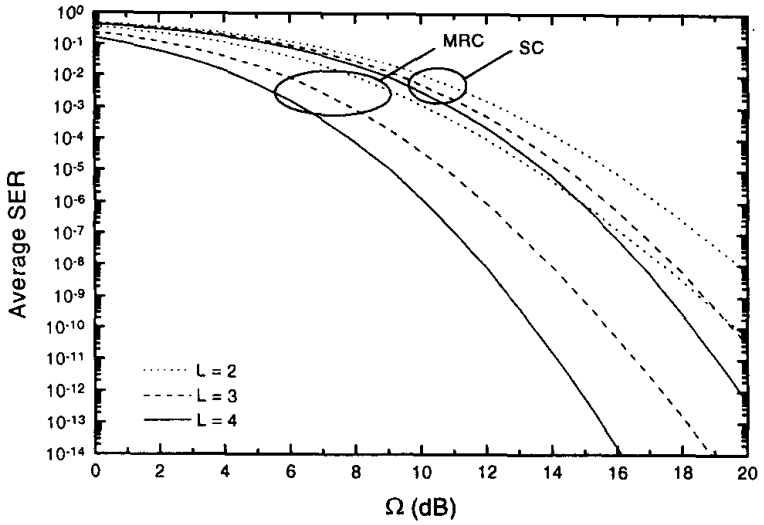

Fig. 1. Average SER (versus $\Omega$ ) of $L$-fold branch $M R C$ and SC for noncoherent MFSK in Nakagami- $m$ fading channel with $m=5$.

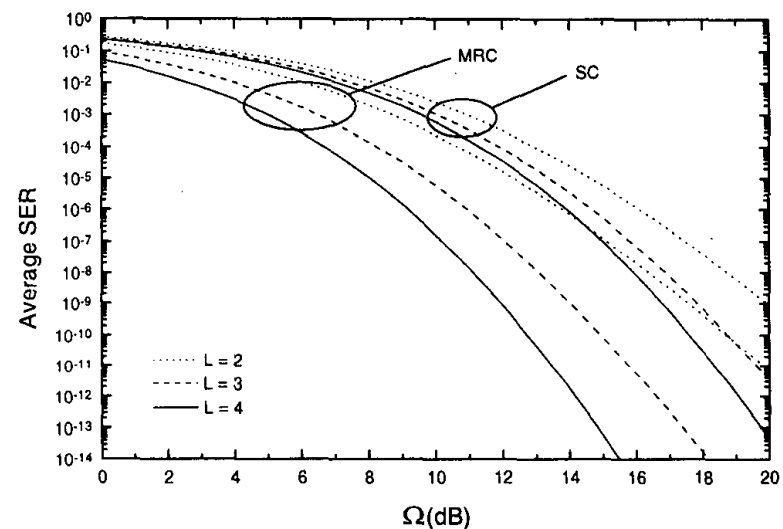

Fig.3. Average SER (versus $\Omega$ ) of $L$-fold branch MRC and SC for MPSK in Nakagami- $m$ fading channel with $m=5$.

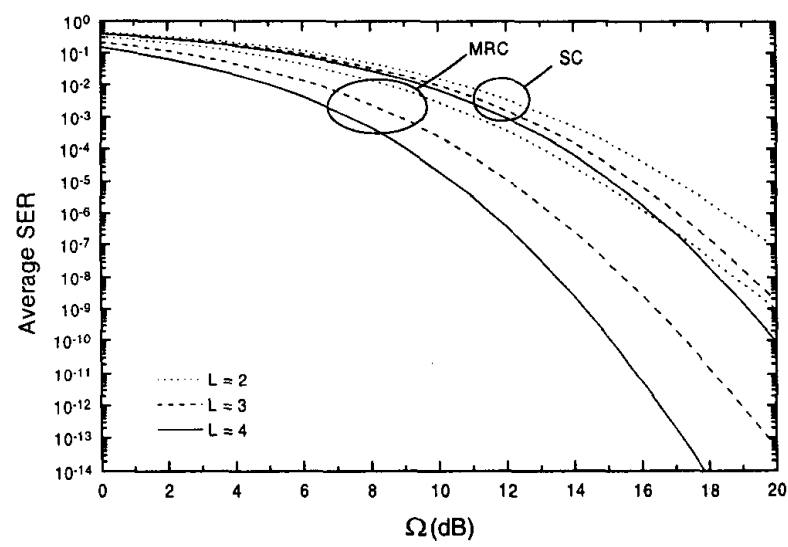

Fig.5. Average SER (versus $\Omega$ ) of $L$-fold branch MRC and SC for MDPSK in Nakagami- $m$ fading channel with $m=5$.

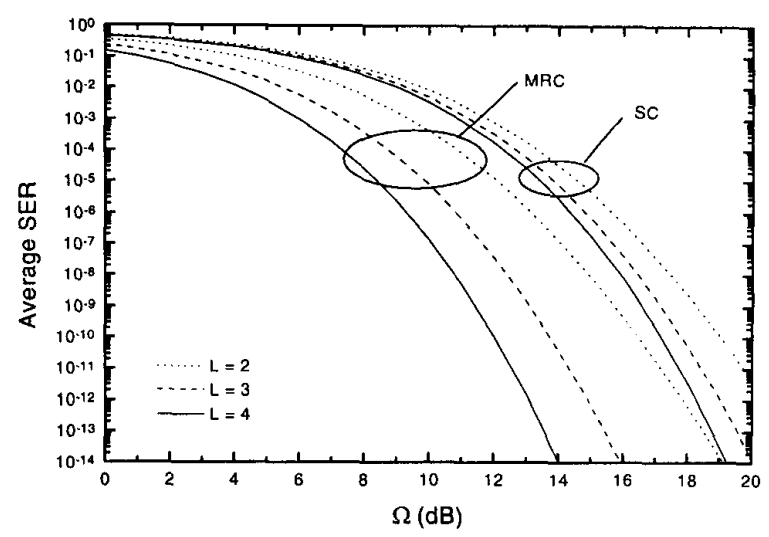

Fig. 2. Average SER (versus $\Omega$ ) of $L$-fold branch MRC and SC for noncoherent MFSK in Nakagami- $m$ fading channel with $m=10$.

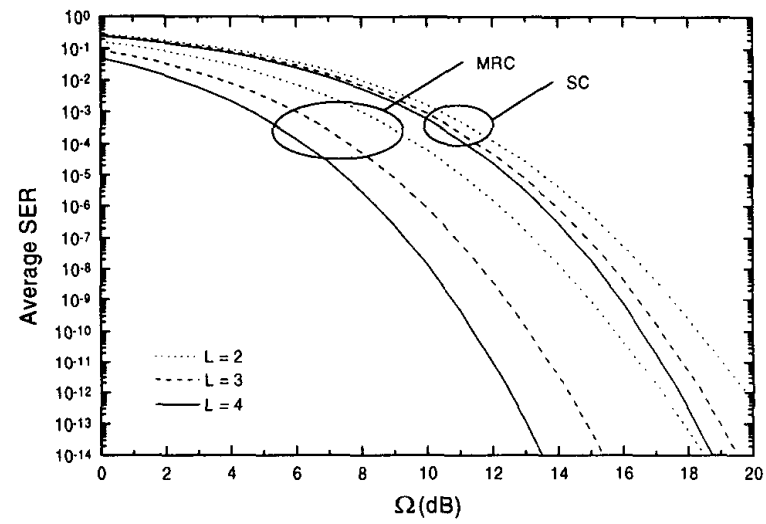

Fig.4. Average SER (versus $\Omega$ ) of $L$-fold branch MRC and SC for MPSK in Nakagami- $m$ fading channel with $m=10$.

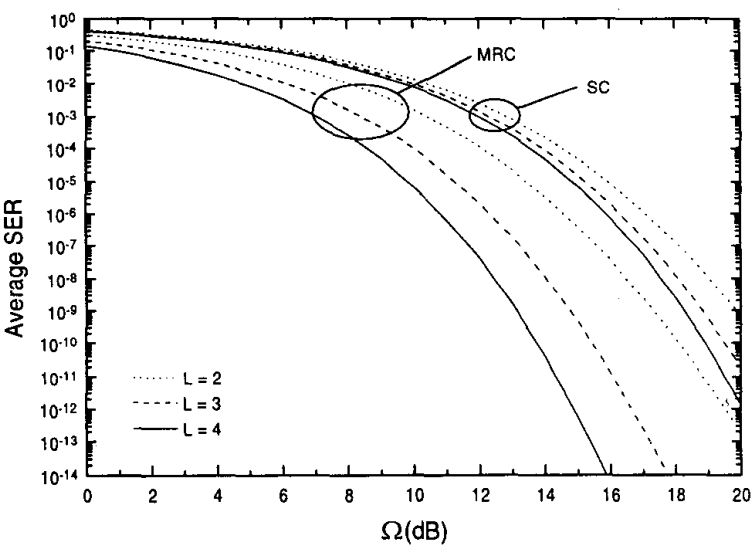

Fig.6. Average SER (versus $\Omega$ ) of $L$-fold branch MRC and SC for MDPSK in Nakagami- $m$ fading channel with $m=10$. 